\title{
EVIDENCE FOR A COMPACT WOLF-RAYET PROGENITOR FOR THE TYPE Ic SUPERNOVA PTF 10vgv
}

\author{
A. Corsi ${ }^{1}$, E. O. Ofek ${ }^{2}$, A. Gal-Yam ${ }^{2}$, D. A. Frail ${ }^{3}$, D. Poznanski ${ }^{4}$, P. A. Mazzali ${ }^{5}, 6$, S. R. Kulkarni ${ }^{7}$, M. M. Kasliwal ${ }^{7}$,

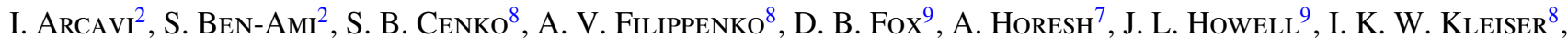

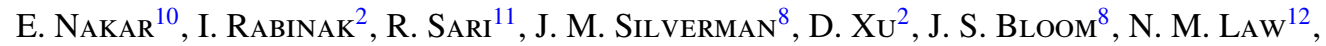 \\ P. E. NugEnT ${ }^{8,13}$, AND R. M. QuimbY ${ }^{7}$ \\ ${ }^{1}$ LIGO laboratory, California Institute of Technology, MS 100-36, Pasadena, CA 91125, USA; corsi@ caltech.edu \\ ${ }^{2}$ Department of Particle Physics and Astrophysics, The Weizmann Institute of Science, Rehovot 76100, Israel \\ ${ }^{3}$ National Radio Astronomy Observatory, P.O. Box 0, Socorro, NM 87801, USA \\ ${ }^{4}$ School of Physics and Astronomy, Tel-Aviv University, Tel-Aviv 69978, Israel \\ ${ }^{5}$ INAF-Osservatorio Astronomico, vicolo dellOsservatorio, 5, I-35122 Padova, Italy \\ ${ }^{6}$ Max-Planck Institut für Astrophysik, Karl-Schwarzschild-Str. 1, D-85748 Garching, Germany \\ ${ }^{7}$ Cahill Center for Astrophysics, California Institute of Technology, Pasadena, CA 91125, USA \\ ${ }^{8}$ Department of Astronomy, University of California, Berkeley, CA 94720-3411, USA \\ ${ }^{9}$ Department of Astronomy \& Astrophysics, Pennsylvania State University, University Park, PA 16802, USA \\ ${ }^{10}$ Raymond and Beverly Sackler School of Physics \& Astronomy, Tel Aviv University, Tel Aviv 69978, Israel \\ ${ }^{11}$ Racah Institute for Physics, The Hebrew University, Jerusalem 91904, Israel \\ 12 Dunlap Institute for Astronomy and Astrophysics, University of Toronto, 50 St. George Street, Toronto, M5S 3H4 Ontario, Canada \\ ${ }^{13}$ Computational Cosmology Center, Lawrence Berkeley National Laboratory, 1 Cyclotron Road, Berkeley, CA 94720, USA \\ Received 2011 October 25; accepted 2012 January 4; published 2012 February 8
}

\begin{abstract}
We present the discovery of PTF 10vgv, a Type Ic supernova (SN) detected by the Palomar Transient Factory, using the Palomar 48 inch telescope (P48). $R$-band observations of the PTF 10vgv field with P48 probe the SN emission from its very early phases (about two weeks before $R$-band maximum) and set limits on its flux in the week prior to the discovery. Our sensitive upper limits and early detections constrain the post-shock-breakout luminosity of this event. Via comparison to numerical (analytical) models, we derive an upper-limit of $R \lesssim 4.5 R_{\odot}\left(R \lesssim 1 R_{\odot}\right)$ on the radius of the progenitor star, a direct indication in favor of a compact Wolf-Rayet star. Applying a similar analysis to the historical observations of SN 1994I yields $R \lesssim 1 / 4 R_{\odot}$ for the progenitor radius of this SN.
\end{abstract}

Key words: supernovae: general - supernovae: individual (PTF 10vgv)

Online-only material: color figures, machine-readable table

\section{INTRODUCTION}

Core-collapse supernovae ( $\mathrm{SNe})$ are believed to originate from evolved, massive progenitors (initial mass $\gtrsim 8-10 M_{\odot}$ ) whose iron core undergoes gravitational collapse. Among them, Type II-Plateau (II-P) SNe show prominent hydrogen in their spectrum and a plateau in the optical light curves. Type IIb SNe have hydrogen in the spectrum initially and an H-deficient spectrum at later times. Finally, Types $\mathrm{Ib}$ and Ic show no evidence for hydrogen at any time. The $\mathrm{H}$-deficient/H-poor core-collapse $\mathrm{SNe}$ are thought to be produced by progenitors stripped of their hydrogen (SN Ib) and possibly helium (SN Ic) envelopes prior to exploding (for a review, see Filippenko 1997). Due to the stiff dependence of mass loss on luminosity/mass, a sequence of increasing main-sequence mass may be pictured going from progenitors of SNe II-P, IIb, Ib, and Ic (Heger et al. 2003; Crowther 2007; Georgy et al. 2009). Rotation, metallicity, and binarity also affect the mass loss (e.g., Podsiadlowski et al. 1992; Meynet et al. 1994; Meynet \& Maeder 2000).

The viability of the standard explosion mechanism for stars of increasing mass is challenging, given that their higher mass cores are more bound, and their SN shocks subject to a very high accretion rate (e.g., Burrows et al. 2007). Binary-star evolution has been studied as a channel to circumvent this caveat (e.g., Utrobin 1994; Woosley et al. 1994; Fryer et al. 2007; Yoon et al. 2010; Smith et al. 2011). The basic ingredient of this scenario is mass loss through transfer onto a companion. In this case, the mass-loss luminosity scaling does not apply, and much lower mass progenitors can explode as H-poor cores.
Recently, Dessart et al. (2011) published simulations of $\mathrm{SN}$ light curves resulting from explosions of $\mathrm{SN} \mathrm{IIb/Ib/Ic}$ progenitors. All SNe show a $\sim 10$ day long post-breakout plateau with a luminosity of $(1-5) \times 10^{7} L_{\odot}$. Analytical estimates for the early-time $(t \lesssim 1-2$ days since explosion) post-breakout emission have been provided by Rabinak \& Waxman (2011) and Nakar \& Sari (2010).

In this Letter, we present the discovery of a type Ic SN, PTF 10vgv, detected by the Palomar Transient Factory ${ }^{14}$ (PTF; Law et al. 2009; Rau et al. 2009) (Section 2). We report its spectral classification (Section 3) and the radio follow-up observations (Section 4). We constrain the radius of the stellar progenitor of this SN by comparing our tight pre-discovery upper-limits with the predictions of several models (Dessart et al. 2011; Rabinak \& Waxman 2011; Nakar \& Sari 2010) (Section 5).

\section{DISCOVERY AND $R$-BAND PHOTOMETRY}

On 2010 September 14.1446 (UTC times are used throughout), we discovered a Type Ic SN, PTF 10vgv, via the automated Oarical software (Bloom et al. 2011). The SN was visible at a magnitude of $R \approx 19.9$ (Table 1 and Figure 1) in an image (60 $\mathrm{s}$ exposure) taken with the Palomar Oschin Schmidt 48 inch telescope (P48). It was not seen in previous images of the same field taken on 2010 September 12.4830, down to a limiting magnitude of $R>20.2$. The SN J2000 position is $\alpha=22^{\mathrm{h}} 16^{\mathrm{m}} 01^{\mathrm{s}} .17$,

\footnotetext{
${ }^{14}$ http://www.astro.caltech.edu/ptf/
} 


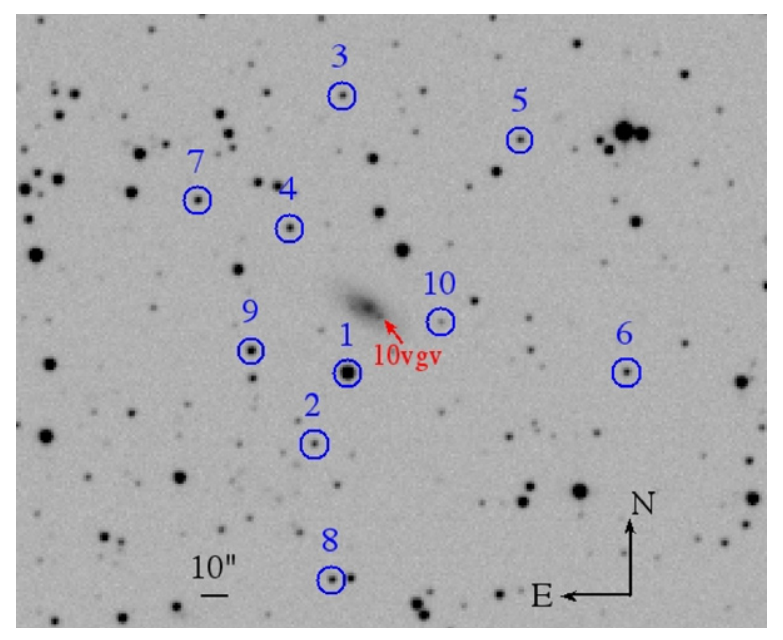

Figure 1. Discovery image of PTF 10vgv (marked with a red arrow) in the $R$ band; the host galaxy is also visible. Circles of $5^{\prime \prime}$ radius mark the positions of the 10 reference stars used for calibration of the P48 photometry (see the text). (A color version of this figure is available in the online journal.)

Table 1

P48 Observations of PTF 10vgv in $R$-band

\begin{tabular}{lcc}
\hline \hline $\begin{array}{l}\text { Start Time } \\
\text { JD-2455453.6446 (days) }\end{array}$ & $\begin{array}{c}\text { Exposure } \\
(\mathrm{s})\end{array}$ & $\begin{array}{l}\mathrm{Mag}^{\mathrm{a}} \\
(\mathrm{mag})\end{array}$ \\
\hline-6.776 & 600 & $<21.2^{\mathrm{b}}$ \\
-6.776 & 60 & $<20.8^{\mathrm{b}}$ \\
-6.732 & 60 & $<21.1^{\mathrm{b}}$ \\
-5.732 & 60 & $<20.9^{\mathrm{b}}$ \\
-5.688 & 60 & $<20.8^{\mathrm{b}}$ \\
-3.811 & 60 & $<20.6^{\mathrm{b}}$ \\
-3.811 & 360 & $<20.8^{\mathrm{b}}$ \\
-3.766 & 60 & $<20.6^{\mathrm{b}}$ \\
-2.814 & 60 & $<20.8^{\mathrm{b}}$ \\
-2.768 & 60 & $<21.4^{\mathrm{b}}$ \\
\hline
\end{tabular}

Notes.

${ }^{a}$ Magnitudes are not corrected for Galactic extinction and are calibrated to the SDSS $r$ (SDSS is estimated to be on the $\mathrm{AB}$ system within $\pm 0.01 \mathrm{mag}$ in the $r$ and $i$ bands).

b $3 \sigma$ upper limit computed by simulating stars at the position of PTF 10vgv to account for the presence of the underlying host galaxy.

(This table is available in its entirety in a machine-readable form in the online journal. A portion is shown here for guidance regarding its form and content.)

$\delta=+40^{\circ} 52^{\prime} 03^{\prime \prime} .3$ (Corsi et al. 2010a), at an angular distance of $\sim 5^{\prime \prime}$ from the galaxy SDSS ${ }^{15}$ J221601.54+405206.5. P48 observations were obtained with the Mould- $R$ filter (Table 1 and Figure 2). A high-quality image produced by stacking several images of the same field was used as a reference and subtracted from the individual images. Photometry was performed with an aperture of $2^{\prime \prime}$ radius relative to the $r$-band magnitudes of 10 SDSS reference stars in the field (Figure 1), applying color corrections (Corsi et al. 2011). Aperture corrections were applied to account for systematic errors as well as errors introduced by the subtraction process (Corsi et al. 2011).

\section{SPECTRAL CLASSIFICATION}

After rapidly identifying PTF 10vgv, we triggered our followup programs (Gal-Yam et al. 2011). On 2010 September 16 and

\footnotetext{
15 Sloan Digital Sky Survey (York et al. 2000).
}
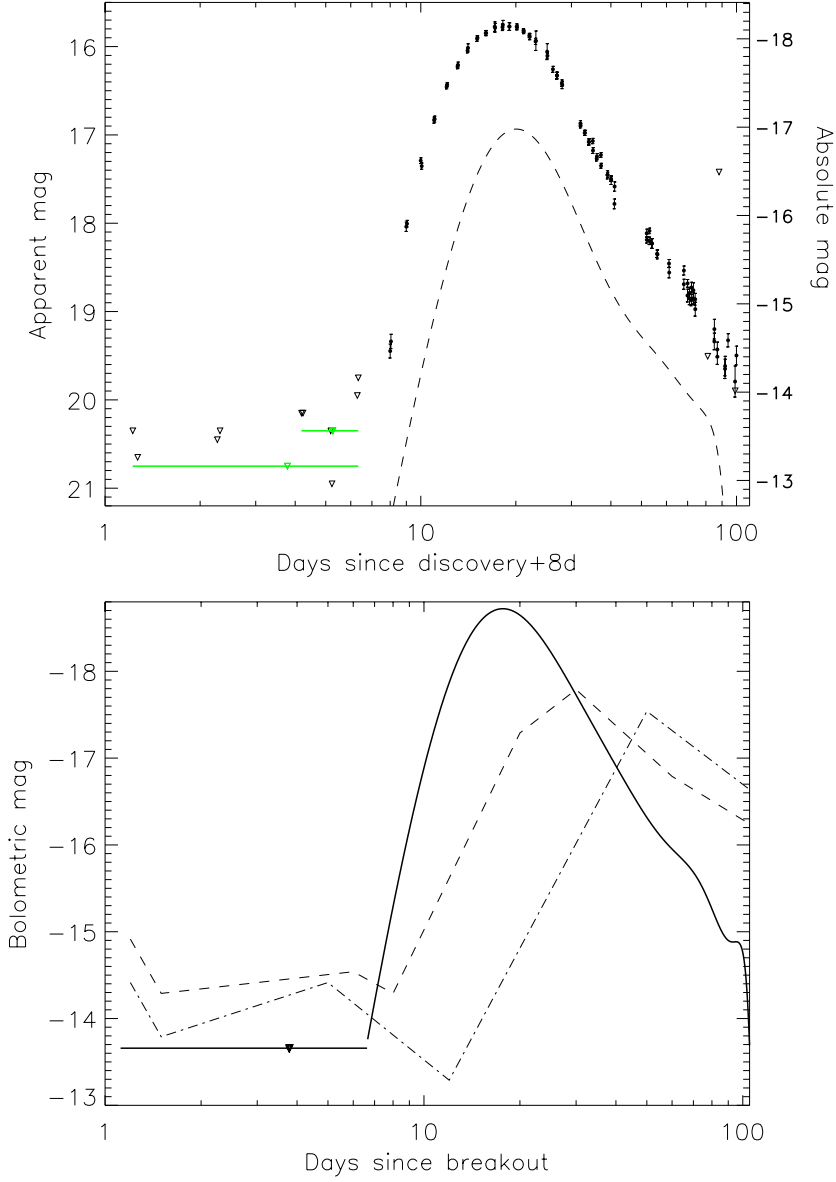

Figure 2. Top: P48 $R$-band light curve of PTF 10vgv (black dots) corrected for Galactic extinction. P48 pre-discovery upper limits derived using $60 \mathrm{~s}$ exposure images are plotted as black triangles. Deeper upper limits obtained by co-adding the pre-explosion images are plotted as green triangles, with the green horizontal lines indicating the time range spanned by the co-added images. For comparison, we also plot the light curve of SN 1994I (dashed line), rescaled to the redshift of PTF 10vgv. Bottom: schematic representations of the bolometric light curves of models Bmi18mf3p79z=1 (dashed line) and Bmi25mf5p09z1 (dash-dotted line) of Dessart et al. (2011) are compared with the PTF 10vgv bolometric light curve (solid line). The black triangle and solid horizontal line indicate our deepest pre-explosion co-added upper limit (see upper panel) rescaled to account for the bolometric correction (and for Galactic extinction). See Section 5 for discussion.

(A color version of this figure is available in the online journal.)

October 1, we observed PTF 10vgv with the dual-arm Kast spectrograph (Miller \& Stone 1993) on the $3 \mathrm{~m}$ Shane telescope at Lick Observatory (Figure 3). We used a $2^{\prime \prime}$ wide slit, a $600 / 4310$ grism on the blue side, and a 300/7500 grating on the red side, yielding full width at half-maximum intensity (FWHM) resolutions of $\sim 4 \AA$ and $\sim 10 \AA$, respectively. All observations were aligned along the parallactic angle to reduce differential light losses (Filippenko 1982). Respective exposure times and air masses were $1800 \mathrm{~s}$ and 1.03 for the first epoch, and $2100 \mathrm{~s}$ and 1.00 for the second epoch. The spectra were reduced using standard techniques (e.g., Foley et al. 2003) based on IRAF and IDL routines. Using the Kast spectra we derive a redshift of $z=0.0142 \pm 0.0002$ (using $\mathrm{H} \beta, \mathrm{O}$ III, $\mathrm{H} \alpha, \mathrm{N}$ II, and $\mathrm{S}$ II lines) for PTF 10vgv.

On 2010 September 27, in between the two epochs of the Kast observations, we observed PTF 10vgv with the Low Resolution Spectrograph (LRS) mounted on the Hobby-Eberly Telescope (HET), using the gr300 grating and GG385 filter. We applied bias- and flat-field corrections using daytime calibration 


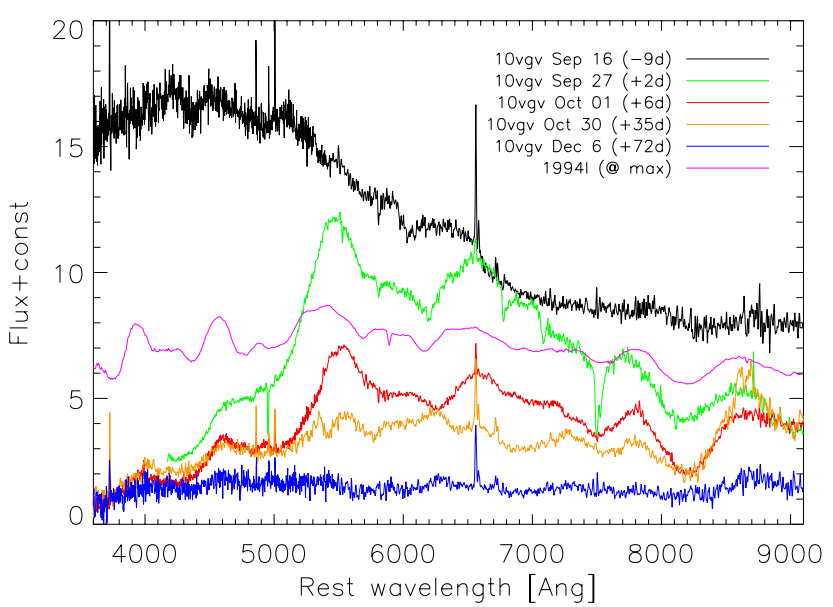

Figure 3. Spectra of PTF 10vgv from Lick/Kast (black and red lines), HET/LRS (green line; telluric absorption lines not removed), and P200/DBSP (orange and blue lines). The approximate epoch since the $R$-band peak is also indicated for each spectrum. For comparison, the spectrum of SN 1994I around maximum light is also shown (magenta). All data are available in digital form from the Weizmann Institute of Science Experimental Astrophysics Spectroscopy System at http://www.weizmann.ac.il/astrophysics/wiseass/.

(A color version of this figure is available in the online journal.)

frames, and removed cosmic rays using the IRAF task "L.A. Cosmic" (van Dokkum 2001). The spectrum was extracted and wavelength-calibrated using the "apall" and "identify" IRAF tasks, respectively, and had exposure time $450 \mathrm{~s}$ at a mean airmass of 1.26.

On 2010 October 30 and December 7, we observed PTF 10vgv with the Double Beam Spectrograph (DBSP; Oke \& Gunn 1982) on the Palomar 200 inch telescope (P200; Figure 3). We used the 600/4000 and the 158/7500 gratings for the blue and red cameras, respectively, with a D55 dichroic, resulting in a spectral coverage of $\sim 3500-9500 \AA$. The spectra were reduced using a custom pipeline combining IRAF and IDL scripts. Respective exposure times and air masses were $600 \mathrm{~s}$ and 1.1 for the first epoch, and $350 \mathrm{~s}$ and 1.04 for the second epoch.

We measured the velocity of the Si II absorption at $6355 \AA$, which traces reasonably closely the position of the photosphere (e.g., Tanaka et al. 2008), using the spectra of PTF 10vgv taken on September 16, September 27, and October 1. The velocities are $16 \times 10^{3} \mathrm{~km} \mathrm{~s}^{-1}, 9 \times 10^{3} \mathrm{~km} \mathrm{~s}^{-1}$, and $6 \times 10^{3} \mathrm{~km} \mathrm{~s}^{-1}$, respectively, for the three epochs. These are comparable to those of the "normal" SN Ic 1994I at similar epochs (Sauer et al. 2006), $\sim 0.7$ times those measured for SN 2006aj (associated with X-ray flash 060218; Mazzali et al. 2006), and smaller than those of the gamma-ray burst (GRB)-associated SN 1998bw (Iwamoto et al. 1998) and SN 2003dh (see Figure 5 in Corsi et al. 2011, and references therein). The broad-line SN Ic 2002ap also showed higher velocities $\left(\gtrsim 16 \times 10^{3} \mathrm{~km} \mathrm{~s}^{-1}\right.$ at $\sim 1$ week after the explosion; Gal-Yam et al. 2002; Mazzali et al. 2002).

We thus classify PTF 10vgv as a normal Type Ic SN. A cursory examination of PTF 10vgv spectra suggests that the blending of lines in this SN is stronger than in both SN 2006aj and SN 1994I, indicating that in PTF $10 \mathrm{vgv}$ there may be significantly more mass at $v \approx 2 \times 10^{4} \mathrm{~km} \mathrm{~s}^{-1}$. In Figure 3 , we compare our spectra of PTF 10vgv with the one of SN 1994I around maximum light.

\section{RADIO FOLLOW-UP OBSERVATIONS}

Starting on 2010 October 7.16, we observed the position of PTF 10vgv (along with the necessary calibrators) with the
Expanded Very Large Array (EVLA; Perley et al. 2009) in its $\mathrm{C}$ configuration, at $4.495 \mathrm{GHz}$ and $7.915 \mathrm{GHz}$, for a total time of 30 minutes (Corsi et al. 2010b). We detected no radio emission from the position of PTF $10 \mathrm{vgv}$, down to $3 \sigma$ limits of $120 \mu \mathrm{Jy}$ at $4.495 \mathrm{GHz}$ and $102 \mu \mathrm{Jy}$ at $7.915 \mathrm{GHz}$. Based on this, we estimate the $5 \mathrm{GHz}$ spectral luminosity of PTF $10 \mathrm{vgv}$ to be $\lesssim 5 \times 10^{26} \mathrm{erg} \mathrm{s}^{-1} \mathrm{~Hz}^{-1}$ or $\sim 100$ times below the radio luminosity of the GRB-associated SN 1998bw (Kulkarni et al. 1998) on a similar timescale. This supports the idea that PTF 10vgv is a normal SN Ic, rather than a GRB-associated $\mathrm{SN}$. We re-observed PTF 10vgv with the EVLA in its BnA configuration starting on 2011 May 12.52, for a total time of $1 \mathrm{hr}$ and at a central frequency of $8.46 \mathrm{GHz}$. No radio sources were detected in the error circle of PTF 10vgv down to a $3 \sigma$ limit of $30 \mu \mathrm{Jy}$. EVLA data were reduced and imaged using the AIPS software package.

\section{DISCUSSION}

The measured peak magnitude of PTF 10vgv (see Table 1) corrected for Galactic extinction $\left(A_{R} \approx 0.45\right.$ mag; Schlegel et al. 1998) gives $M_{R}=-18.16 \pm 0.05 \mathrm{mag}$. The peak absolute magnitude of SN 1994I was $M_{R}=-17.99 \pm 0.48$ (Richmond et al. 1996), while SN 2006aj had $M_{R}=-18.81 \pm 0.06 \mathrm{mag}$ (Mazzali et al. 2006). Since PTF 10vgv is intermediate, in terms of $R$-band peak luminosity, between SN 1994I and SN 2006aj, we estimate its nickel mass $M_{56} \mathrm{Ni}, 10 \mathrm{vgv}$ by interpolating between these two SNe (Sauer et al. 2006; Mazzali et al. 2006), using the scaling $L_{\text {peak }} \propto M_{\mathrm{Ni}} \tau_{c}^{-1}$ for the peak luminosity (where $\tau_{c}$ is the light-curve peak width; Arnett 1982) and considering that the PTF 10vgv light curve is a factor of $\sim 1.25$ broader than that of SN 1994I (while we take the same $\tau_{c}$ for PTF 10vgv and SN 2006aj). This yields $M_{56} \mathrm{Ni}_{10 \mathrm{vgv}} \approx 0.12 M_{\odot}$.

The mass and kinetic energy of the SN ejecta scale as (Arnett 1982) $M_{\mathrm{ej}} \propto \tau_{c}^{2} v_{\mathrm{ph}}$ and $E_{\mathrm{K}} \propto \tau_{c}^{2} v_{\mathrm{ph}}^{3}$, where $v_{\mathrm{ph}}$ is the photospheric velocity. Using these scalings, and considering that the photospheric velocities of PTF $10 \mathrm{vgv}$ are comparable to those of SN 1994I and $\sim 0.7$ times those of SN 2006aj (Section 3), we estimate the ejecta mass and kinetic energy of PTF 10vgv interpolating between SN 2006aj (Mazzali et al. 2006) and SN 1994I (Sauer et al. 2006). We get $M_{\mathrm{ej}, 10 \mathrm{vgv}}=$ $(1.5 \pm 0.3) M_{\odot}$ and $E_{\mathrm{K}, 10 \mathrm{vgv}}=(0.9 \pm 0.3) \times 10^{51} \mathrm{erg}$. This estimate may be refined through spectral modeling. Our spectral analysis suggests that a different mass-velocity distribution may be realized in PTF 10vgv (Section 3), which may lead to a larger $E_{\mathrm{K}}$ than estimated on the basis of the light-curve properties, since the latter are mostly determined by the opacity in the inner ejecta.

Our pre-discovery upper limits can be used to constrain the radius of the stellar progenitor of PTF $10 \mathrm{vgv}$ via comparison with model predictions (Dessart et al. 2011; Rabinak \& Waxman 2011; Nakar \& Sari 2010). We apply a bolometric correction to our $R$-band data, computed assuming that the $\mathrm{SN}$ emits as a black body at temperature $T_{\text {phot }}$ (and neglecting redshift corrections):

$$
\begin{aligned}
M_{\mathrm{bol}}-M_{R}= & -2.5 \log _{10}\left(\frac{4 \pi(10 \mathrm{pc})^{2} F_{0} \int_{\nu_{1}}^{\nu_{2}} S(\nu) d \nu}{L_{\odot}}\right)+M_{\mathrm{bol}, \odot} \\
& +2.5 \log _{10}\left(\frac{\int_{\nu_{1} / k T}^{\nu_{2} / k T} S(x) x^{3}\left(e^{x}-1\right)^{-1} d x}{\pi^{4} / 15}\right), \quad \text { (1) }
\end{aligned}
$$

where $M_{\mathrm{bol}, \odot}=4.72 ; S(v)$ is the P48 Mould- $R$ filter transmission; $v_{1}-v_{2}=(4.1-5.3) \times 10^{14} \mathrm{~Hz}$; and $F_{0}$ is the photometric 
zero-point flux $\left(F_{0}=3.631 \times 10^{-20} \mathrm{erg} \mathrm{cm}^{-2} \mathrm{~s}^{-1} \mathrm{~Hz}^{-1}\right.$ for AB magnitudes). We conservatively maximize the bolometric correction setting $T_{\text {phot }} \approx 10^{4} \mathrm{~K}$, the largest early-time ( $t \lesssim 10$ days since breakout) temperature predicted by the ${ }^{56} \mathrm{Ni}$-rich models of Dessart et al. (2011; see their Figure 2, bottom-left panel). In this way we get $M_{\mathrm{bol}}-M_{R}=-0.496 \mathrm{mag}$.

The optical luminosity of core-collapse SNe after breakout depends on the ejecta composition (via the opacity parameter), the stellar radius, and the $E_{\mathrm{K}} / M_{\mathrm{ej}}$ ratio. A larger $E_{\mathrm{K}} / M_{\mathrm{ej}}$ ratio and a lower $\mathrm{He}$ fraction both increase the predicted luminosity, for a given stellar radius (Rabinak \& Waxman 2011, Equations (25) and (29)).

In recent numerical simulations of core-collapse explosions of single and binary progenitors of $\mathrm{SNe} \mathrm{IIb} / \mathrm{Ib} / \mathrm{Ic}$, Dessart et al. (2011) predicted the existence a $\sim 10$ day long $(\sim 10$ times shorter than in SNe II-P) post-breakout ${ }^{16}$ plateau, with a luminosity of $(1-5) \times 10^{7} L_{\odot}(\sim 10$ times smaller than in $\mathrm{SNe}$ II-P). This plateau has the same origin as that observed in $\mathrm{SNe}$ II-P, ${ }^{17}$ but in the case of $\mathrm{SNe} \mathrm{IIb} / \mathrm{Ib} / \mathrm{Ic}$ it is predicted to have a smaller duration and luminosity because of a more compact progenitor.

For PTF 10vgv we can exclude the presence of a postbreakout plateau with luminosity greater than the one of the compact progenitor model Bmi25mf5p09z1 (Figure 2, lower panel). We thus derive $R \lesssim 4.4 R_{\odot}$ for the radius of PTF $10 \mathrm{vgv}$ progenitor. However, the stellar models analyzed by Dessart et al. (2011) have $E_{\mathrm{K}} / M_{\mathrm{ej}}$ lower than we derive here, and a high surface He fraction. So, the bound on the progenitor radius derived from the comparison with these models is likely overestimated.

Similar limits $\left(R \lesssim 5 R_{\odot}\right)$ can be derived using the predictions by Nakar \& Sari (2010; black line in their Figure 3). But this model is accurate only up to $\lesssim 11 \mathrm{hr}$ after the explosion, since recombination is not treated.

Using $M_{\mathrm{ej}, 10 \mathrm{vgv}}=(1.5 \pm 0.3) M_{\odot}$ and $E_{\mathrm{K}, 10 \mathrm{vgv}}=(0.9 \pm$ $0.3) \times 10^{51} \mathrm{erg}$, as derived above, the tightest constraint, $R \lesssim 0.7 R_{\odot}$, is obtained from the $\mathrm{C} / \mathrm{O}$ model of Rabinak \& Waxman (2011) that accounts for the dependence of the opacity on the envelope composition. The same model, for an envelope composed of mostly He, gives us $R \lesssim 1.3 R_{\odot}$. Thus, $R \lesssim 1 R_{\odot}$ is a reasonable estimate (considering that progenitors of type Ic $\mathrm{SNe}$ may contain a small fraction of $\mathrm{He}$ in the outer layers; Georgy et al. 2009).

Applying this same analytical model to the first clear detection of SN 1994I (Sauer et al. 2006, Figure 8; Richmond et al. 1996, Figure 7), we get $R \lesssim 1 / 4 R_{\odot}$, considering that $M_{\mathrm{ej}, 1994 \mathrm{I}} \approx$ $M_{\mathrm{ej}, 10 \mathrm{vgv}}, E_{\mathrm{K}, 1994 \mathrm{I}} \approx E_{\mathrm{K}, 10 \mathrm{vgv}}$, and that the luminosity of SN 1994I at the time of detection was $\approx 3$ times smaller than the one of PTF $10 \mathrm{vgv}$.

Our limits for PTF $10 \mathrm{vgv}, R \lesssim(1-5) R_{\odot}$, are consistent with a small Wolf-Rayet star (e.g., Crowther 2007), as expected for a highly stripped SN Ic. Almost all Galactic WN stars with

\footnotetext{
16 The breakout of a shock through the stellar surface is predicted to be the first electromagnetic signal marking the birth of a SN (e.g., Falk \& Arnett 1977; Falk 1978; Klein \& Chevalier 1978; Chevalier 1992; Waxman et al. 2007; Nakar \& Sari 2010; Rabinak \& Waxman 2011).

17 The plateau is associated with a cooling and recombination wave (CRW) propagating downward through the SN envelope, separating almost recombined outer layers from strongly ionized inner ones (e.g., Nadyozhin 2003). During the plateau phase, the photosphere sits on the upper edge of the CRW front, whose downward speed is approximately equal to the outward expansion velocity, thus $R_{\text {phot }} \approx$ const. Since also $T_{\text {phot }} \approx$ const $\approx T_{\text {recomb }}$ (where $T_{\text {recomb }}$ is the recombination temperature), a plateau in the luminosity is expected.
}

hydrogen (WNL; e.g., Hamann et al. 2006) have $R \gtrsim 5 R_{\odot}$, and all of those reported there have $R \gtrsim 2 R_{\odot}$. Our result thus favors a progenitor having no hydrogen at the surface (WNE, WC, or WO, e.g., Sander et al. 2011), in agreement with the fact that Ic $\mathrm{SN}$ progenitors are generally thought to be stripped of their H- (and He-) rich layers (e.g., Gal-Yam et al. 2005; Smartt 2009).

PTF 10vgv provides the first constraint on the progenitor radius of an SN ever obtained from optical pre-explosion limits extending up to a week before discovery. Optical surveys with rapid cadence and relatively deep exposures (like PTF) should allow us to study many more objects in this manner.

We thank Boaz Katz and Eli Waxman for useful comments. PTF is a collaboration of Caltech, LCOGT, the Weizmann Institute, LBNL, Oxford, Columbia, IPAC, and UC Berkeley. Staff and computational resources were provided by NERSC, supported by the DOE Office of Science. Lick Observatory and the Kast spectrograph are operated by the University of California. HET and its LRS are supported by UT Austin, the Pennsylvania State University, Stanford, Ludwig-MaximiliansUniversität München, Georg-August-Universität Göttingen, and the Instituto de Astronomia de la Universidad Nacional Autonoma de Mexico. The EVLA is operated by NRAO for the NSF, under cooperative agreement by Associated Universities, Inc. We thank the staffs of the above observatories for their assistance. A.G. and S.R.K. acknowledge support from the BSF; A.G. further acknowledges support from the ISF, FP7/IRG, Minerva, the Sieff Foundation, and the German-Israeli Fund (GIF). A.V.F. and his group at UC Berkeley acknowledge generous financial assistance from Gary \& Cynthia Bengier, the Richard \& Rhoda Goldman Fund, the TABASGO Foundation, and NSF grant AST-0908886. A.C. acknowledges support from LIGO, which was constructed by Caltech and MIT with funding from the NSF under cooperative agreement PHY-0757058, and partial support from NASA/Swift grant NNH10ZDA001N.

\section{REFERENCES}

Arnett, W. D. 1982, ApJ, 253, 785

Bloom, J. S., Richards, J. W., Nugent, P. E., et al. 2011, PASP, submitted (arXiv:1106.5491)

Burrows, A., Livne, E., Dessart, L., Ott, C. D., \& Murphy, J. 2007, ApJ, 655, 416

Chevalier, R. A. 1992, ApJ, 394, 599

Corsi, A., Cenko, S. B., Ofek, E. O., et al. 2010a, ATel, 2914, 1

Corsi, A., Ofek, E. O., Frail, D. A., et al. 2011, ApJ, 741, 76

Corsi, A., Ofek, E. O., Frail, D. A., et al. 2010b, ATel, 2915, 1

Crowther, P. A. 2007, ARA\&A, 45, 177

Dessart, L., Hillier, D. J., Livne, E., et al. 2011, MNRAS, 414, 2985

Falk, S. W., \& Arnett, W. D. 1977, ApJS, 33, 515

Falk, S. W. 1978, ApJ, 225, 133

Filippenko, A. V. 1982, PASP, 94, 715

Filippenko, A. V. 1997, ARA\&A, 35, 309

Foley, R. J., Papenkova, M. S., Swift, B. J., et al. 2003, PASP, 115, 1220

Fryer, C. L., Mazzali, P. A., Prochaska, J., et al. 2007, PASP, 119, 1211

Gal-Yam, A., Kasliwal, M. M., Arcavi, I., et al. 2011, ApJ, 736, 159

Gal-Yam, A., Ofek, E. O., \& Shemmer, O. 2002, MNRAS, 332, L73

Gal-Yam, A., Fox, D. B., Kulkarni, S. R., et al. 2005, ApJ, 630, L29

Georgy, C., Meynet, G., Walder, R., Folini, D., \& Maeder, A. 2009, A\&A, 502, 611

Hamann, W.-R., Gräfener, G., \& Liermann, A. 2006, A\&A, 457, 1015

Heger, A., Fryer, C. L., Woosley, S. E., Langer, N., \& Hartmann, D. H. 2003, ApJ, 591,288

Iwamoto, K., Mazzali, P. A., Nomoto, K., et al. 1998, Nature, 395, 672

Klein, R. I., \& Chevalier, R. A. 1978, ApJ, 223, L109

Kulkarni, S. R., Frail, D. A., Wieringa, M. H., et al. 1998, Nature, 395, 663 
Law, N. M., Kulkarni, S. R., Dekany, R. G., et al. 2009, PASP, 121, 1395

Mazzali, P. A., Deng, J., Maeda, K., et al. 2002, ApJ, 572, L61

Mazzali, P. A., Deng, J., Nomoto, K., et al. 2006, Nature, 442, 1018

Meynet, G., \& Maeder, A. 2000, A\&A, 361, 101

Meynet, G., Maeder, A., Schaller, G., Schaerer, D., \& Charbonnel, C. 1994, A\&AS, 103, 97

Miller, J. S., \& Stone, R. P. S. 1993, Lick Observatory Technical Report 66 (Santa Cruz, CA: Lick Observatory)

Nadyozhin, D. K. 2003, MNRAS, 346, 97

Nakar, E., \& Sari, R. 2010, ApJ, 725, 904

Oke, J. B., \& Gunn, J. E. 1982, PASP, 94, 586

Perley, R., Napier, P., Jackson, J., et al. 2009, IEEE Proc., 97, 1448

Podsiadlowski, P., Joss, P. C., \& Hsu, J. J. L. 1992, ApJ, 391, 246

Rabinak, I., \& Waxman, E. 2011, ApJ, 728, 63
Rau, A., Kulkarni, S. R., Law, N. M., et al. 2009, PASP, 121, 1334

Richmond, M. W., van Dyk, S. D., Ho, W., et al. 1996, AJ, 111, 327

Sander, A., Hamann, W.-R., \& Todt, H. 2011, BSRSL, 80, 185

Sauer, D. N., Mazzali, P. A., Deng, J., et al. 2006, MNRAS, 369, 1939

Schlegel, D. J., Finkbeiner, D. P., \& Davis, M. 1998, ApJ, 500, 525

Smartt, S. J. 2009, ARA\&A, 47, 63

Smith, N., Li, W., Filippenko, A. V., \& Chornock, R. 2011, MNRAS, 412, 1522

Tanaka, M., Mazzali, P. A., Benetti, S., et al. 2008, ApJ, 677, 448

Utrobin, V. 1994, A\&A, 281, L89

van Dokkum, P. G. 2001, PASP, 113, 1420

Waxman, E., Mészáros, P., \& Campana, S. 2007, ApJ, 667, 351

Woosley, S. E., Eastman, R. G., Weaver, T. A., \& Pinto, P. A. 1994, ApJ, 429, 300

Yoon, S.-C., Woosley, S. E., \& Langer, N. 2010, ApJ, 725, 940

York, D. G., Adelman, J., Anderson, J. E. Jr, et al. 2000, AJ, 120, 1579 